\title{
Del lado de allá y del lado de acá. ¿Qué pueden aprender mutuamente los países emergentes latinoamericanos y España para mejorar sus perfiles del médico de familia?
}

\author{
Brenda Beatriz Báez-Montiel, Elizabeth Gutiérrez-Islas, Margarita Bolaños-Maldonado, \\ Elena Samarín-Ocampos, José Luis Turabián Fernández
}

Centro de Salud "Santa $M^{a}$ de Benquerencia". Polígono Industrial. Toledo.

Dirección para correspondencia: José Luis Turabián. C/ Calderón de la Barca, 24. 45313 Yepes, Toledo. Correo electrónico: jurabianf@meditex.es

Recibido el 13 de octubre de 2013.

Aceptado para su publicación el 30 de enero de 2014.

\section{RESUMEN}

Objetivo: Se realizó un estudio cualitativo con el objetivo de encontrar factores estratégicos en medicina de familia que impliquen oportunidades de aprendizaje que los países del Norte desarrollado puedan utilizar de los países del Sur emergentes, y viceversa.

Diseño, emplazamiento y participantes: A partir de un muestreo no aleatorio, intencionado, seguido de muestra en bola de nieve, hasta la saturación de los datos, se realizaron por parte médicos de familia de atención primaria de Toledo y Tenerife en España, y Paraguay, México, y Perú, diarios de campo de un día habitual y su contexto socio-sanitario, y se evaluaron mediante análisis del contenido y un estudio DAFO (Debilidades, Amenazas, Fortalezas y Oportunidades). Mediciones principales y resultados: Se obtuvieron 24 diarios de un día normal (9 médicos en España, 7 en México, 4 en Paraguay, y 4 en Perú). Se encontraron "fortalezas" del Sur en la coexistencia de trabajo ambulatorio y hospitalario, en el amplio rango de tareas asistenciales, en el mayor peso de actividades de promoción y educación para la salud dirigidas a la población, en la presencia de actividades comunitarias de campo, en la integración de la medicina tradicional, en la mejor relación médico-paciente, y percepción más positiva de la carga de trabajo. Y "fortalezas" del Norte en las actividades de prevención, promoción y educación para la salud integradas en la asistencia por enfermedades en el centro de salud, y en la mayor existencia de recursos materiales.

Conclusiones: Hay áreas donde podría aprender el médico de familia español del latinoamericano y viceversa.

PALABRAS CLAVE: Medicina de Familia; Educación Basada en Competencias; Investigación Cualitativa; Intercambio Educacional Internacional; Competencia Profesional; Sistemas de Salud.

\section{ABSTRACT}

From one side of the ocean to the other. What can emerging latinamerican countries and Spain learn from each other to improve their family physician profile?

Objective: A qualitative study was carried out with the aim to find strategic factors involving learning opportunities that northern developed countries could use from emerging countries in the South and vice versa.

Design, setting and participants: A non-random intentional sampling, followed by a snowball sampling until data saturation were carried out by a group of primary health care family physicians in Toledo and Tenerife in Spain and Paraguay, Mexico and Peru. Field diaries of a normal day and its health and social care context were kept and analyzed, and a SWOT study (strengths weaknesses, opportunities and threats) was performed.

Main measures and results: Twenty-four diaries of a normal day were obtained (9 physicians in Spain, 7 in Mexico, 4 in Paraguay and 4 in Peru). Some strengths were found in the South in the coexistence of ambulatory care and hospital work, in the wide range of care tasks, in the greater weight of health promotion and educational activities targeted to the population, in the presence of community field activities, in the integration of traditional medicine, in the better patient-doctor relationship and a more positive perception of the workload. And the strengths in northern countries were: more prevention, health promotion and educational actions integrated in visits to the family physician in primary care settings and more material resources available.

Conclusions: There are some areas in which the Spanish family physician could learn from the latinamerican physician and vice versa.

KEYWORDS: Family Practice; Competency-Based Education; Qualitative Research; International Educational Exchange; Professional Competence; Health Systems. 


\section{INTRODUCCIÓN}

Los médicos de familia (MdF) son generalistas capacitados, a nivel de postgrado, para hacer frente a la mayoría de las necesidades de atención primaria (AP), de los pacientes de todas las edades en las comunidades a las que sirven, y se acepta la exigencia de lograr su mejor preparación para resolver las influencias globales en un mundo interdependiente, pidiéndose a los educadores clínicos de medicina de familia (MF) el desarrollo de redes de formación e investigación a nivel internacional ${ }^{1-4}$.

Se acepta que hay un "Norte" con países ricos y un "Sur" con países menos desarrollados o emergentes. Esta división origina relaciones asimétricas con una dominación intelectual del Norte sobre el Sur y, como consecuencia, es difícil la colaboración científica honesta entre iguales. De esta forma, parece que la MF sólo puede aprender de personas, experiencias y contextos inscritos en los países desarrollados, y ha habido una devaluación sistemática de todo lo que fuera procedente de los no desarrollados, faltando un verdadero esfuerzo para profundizar en sus formas de hacer las cosas y experiencia y, en consecuencia, los logros de la medicina latinoamericana permanecen poco conocidos en el mundo. Sin embargo, el "Sur" no sólo recibe ideas, sino que las genera también ${ }^{5,6}$.

En esta situación, faltan estudios comparativos del perfil profesional del MdF -organización del trabajo, actividades, competencias y responsabilidades, satisfacción profesional, relación médico-paciente, etc.- en países industrializadas y emergentes, siendo preciso favorecer la investigación y formación como procesos de doble vía, así como la evaluación adecuada de las fortalezas de los sistemas sanitarios de los países en desarrollo y las redes de colaboración entre países en desarrollo y desarrollados $^{7-9}$.

Por otra parte, hay un creciente interés por los métodos cualitativos en MF ya que proporcionan visiones profundas de los problemas, y dentro de ellos el análisis DAFO ("strengths, weaknesses, opportunities y threats"; en castellano y cambiando el orden de las siglas: debilidades, amenazas, fortalezas y oportunidades) es uno de los métodos usados en el diagnóstico y planificación estratégica, incluida la sanitaria, más simple y difundido. Sin embargo, son menos las investigaciones sobre el empleo práctico y adecuación de este instrumento para aspectos académicos y formativos, especialmente en relación al perfil profesional del MdF en $\mathrm{AP}^{10-12}$. En este contexto, realizamos un estudio cualitativo del perfil profesional, mediante la narración de un diario de un día normal de trabajo de MdF de Paraguay, México, Perú y España, y analizamos los resultados con matrices DAFO para señalar los rum- bos estratégicos de aprendizaje que el Sur puede realizar del Norte y viceversa, para la mejora mutua.

\section{PARTICIPANTES Y MÉTODOS}

El estudio se realizó desde mayo a diciembre de 2012. Se trata de un estudio cualitativo, observacional, narrativo, de elaboración de diarios de campo de un día normal de trabajo, y valoración de los mismos mediante análisis del contenido, y la realización de una matriz DAFO. Este estudio corresponde a la segunda parte de otro mayor, comparativo de los perfiles profesionales de los MdF de diversos países Latinoamericanos (Paraguay, México, y Perú) y de España ${ }^{13}$.

Se efectuó un muestreo no aleatorio, intencionado -de conveniencia- por parte de los investigadores, seguido de muestra en bola de nieve. El procedimiento fue así: los investigadores, que eran médicos residentes de Medicina de Familia y Comunitaria en Toledo (España), y procedían además de España, de Paraguay, México, y Perú, contactaron con MdF de sus países que se incluirían en la muestra, y a los que se les pidió que realizasen el diario. A estos participantes se les pidió que localizaran otros con características análogas y les solicitaran sucesivamente realizar el diario, así hasta la saturación de los datos. El número de la muestra fue de 24: 9 médicos en España (2 en Tenerife y 7 en Toledo), 7 en México, 4 en Paraguay, y 4 en Perú. Se consideró un número muestral adecuado cuando se produjo saturación, es decir, que no se obtenían datos nuevos ${ }^{13}$.

Se realizó un análisis del contenido de los diarios definiendo códigos o categorías de los datos cualitativos: relacionando secciones de los datos con categorías, que los investigadores habían desarrollado previamente (plasmadas en las secciones de guión del diario) o desarrolladas durante el proceso de recogida de narraciones, y realizándose este proceso de organización de los datos cualitativos usando ficheros de Microsoft $₫$ Word. Como técnicas para controlar posibles sesgos en la recogida de datos y en el análisis, se utilizaron la verificación y la triangulación. Para la verificación, los resultados e interpretaciones fueron devueltos a los participantes con el fin de comprobar si se sentían representados en ellos. La triangulación se realizó por una parte entre los resultados obtenidos con los datos que se habían comunicado en la literatura de cada país en relación al contexto socio-sanitario y la organización de la atención primaria, y por otra se realizó una búsqueda con la herramienta Google $₫$ en Internet de los términos "Diario de médico" y "Un día normal del médico", para localizar diarios, blogs, artículos sobre diarios, y vídeos del día 
normal, para España, de MdF y que fueron comparados con los obtenidos por los participantes españoles del estudio. De esta forma se obtuvieron distintas perspectivas del fenómeno estudiado utilizando diferentes métodos de investigación ${ }^{13}$.

Finalmente, se realizó un análisis $\mathrm{DAFO}^{11}$ a partir de las categorías o códigos identificados en el análisis del contenido de los diarios, construyéndose una Matriz DAFO para el Sur y otra para el Norte, y se definieron las estrategias de mejora del Norte por los posibles aportes del Sur, y viceversa.

\section{RESULTADOS}

Se obtuvieron 24 diarios: nueve médicos en España (seis varones y tres mujeres; rango de edades: 30-58 años; uno de zonas rurales), siete en México (cuatro varones y tres mujeres; rango de edades: 25-43 años; uno de zona rural), cuatro en Paraguay (dos varones y dos mujeres; rango de edades 39-
56 años; dos de zona rural), y cuatro en Perú (dos varones y dos mujeres; rango de edades: 35-40 años, tres de zona rural).

Se construyó una Matriz DAFO para el "Sur" (Paraguay, México y Perú) [tabla 1] y otra para el Norte (España) [tabla 2]. La matriz DAFO permite visualizar y resumir la situación actual del perfil profesional del MdF en ambas zonas geográficas. Se encontraron "fortalezas" del Sur (Paraguay, México y Perú) en la coexistencia de trabajo ambulatorio y hospitalario, en el amplio rango de tareas asistenciales del médico de familia, incluidas las hospitalarias, en el mayor peso de actividades de prevención, promoción y educación para la salud dirigidas a la población y fuera del centro de salud, en la presencia de actividades comunitarias de campo, en la integración de la medicina tradicional, en la mejor relación médico-paciente, y en la mayor satisfacción laboral y percepción más positiva de la carga de trabajo. Y se encontraron "fortalezas" del Norte (España) en las actividades de prevención, promoción y educación para la salud integradas

\section{DEBILIDADES}

- Gran carga de trabajo por largas jornadas y pluriempleo

- Carencia de recursos sanitarios

- Práctica clínica más relacionada con el hospital

- Poca experiencia en asistencia a enfermedades crónicas no transmisibles

\section{ANÁLISIS INTERNO}

\section{AMENAZAS}

- Contextos muy diferentes pero hostiles y difíciles en general

- Población de bajo nivel socioeconómico, multiétnica, multicultural y resistente a los cambios

- Difícil accesibilidad en zonas rurales

- Trabajo agreste y duro

\section{OPORTUNIDADES}

\section{ANÁLISIS EXTERNO}

- Coexistencia de trabajo ambulatorio y hospital

- Amplio rango de tareas asistenciales del médico de familia - pediatría, geriatría, partos, odontología

- Tareas hospitalarias

- Prevención y educación dirigidas a la población y realizadas fuera del Centro de Salud

- Presencia de medicina tradicional

- Una cosmovisión indígena que promueve una nueva forma de entender el desarrollo, basada en la armonía entre individuos y de éstos con la naturaleza

- Actividades comunitarias

- Satisfacción laboral

Tabla 1. Matriz DAFO para el Sur (Latinoamérica: Paraguay, México y Perú) 


\section{DEBILIDADES}

- Percepción negativa de la carga de demanda

- Rango reducido de tareas asistenciales del médico de familia

- Poca experiencia en asistencia de enfermedades transmisibles

- Situación laboral vivida como incierta

- Insatisfacción laboral

ANÁLISIS - Dificultades en la relación INTERNO médico-paciente

\section{AMENAZAS}

Contextos muy diferentes pero hostiles y difíciles en general. Población autóctona pero con emigrantes, alta tasa de paro, demandante y maleducada

\section{FORTALEZAS}

- Prevención, promoción y educación integradas en la asistencia en el Centro de Salud

- Práctica clínica más liberada del hospital

\section{OPORTUNIDADES}

- Sin carencia de recursos

Tabla 2. Matriz DAFO para el Norte (España)

en la asistencia por enfermedades en el centro de salud, y en la mayor existencia de recursos materiales.

El análisis DAFO mostró cómo estos temas podrían combinarse y transformarse en ciertas estrategias: defensiva, de supervivencia, ofensiva (a partir de las fortalezas) y de reorientación (las oportunidades a aprovechar), para Latinoamérica y para España. España podría aprender del amplio rango de actividades del MdF latinoamericano, del trabajo hospitalario del MdF, de la asistencia a enfermedades transmisibles, de una cosmovisión indígena que promueve una nueva forma de entender el desarrollo basada en la armonía entre los individuos y de éstos con la Naturaleza, de las actividades comunitarias fuera del centro de salud, de la relación médico-paciente, de la satisfacción laboral a pesar de la carencia de recursos, y de la percepción más positiva de la carga de trabajo. Latinoamérica podría aprender a integrar algunas actividades de prevención, promoción, educación para la salud y comunidad en la asistencia en el centro de salud para reducir las dificultades de las largas jornadas de trabajo, especialmente en las zonas rurales más agrestes y duras, de la asistencia a enfermedades crónicas no transmisibles, en la gestión de la asistencia rural, a realizar una práctica clínica más liberada de la hospitalaria, y a evitar el pluriempleo. Así mismo, la carencia de recursos puede dar lugar a una estrategia de intercambios académicos entre los países (tablas 3, 4 y 5).

\section{DISCUSIÓN}

La vida es un proceso de aprendizaje. Aprendemos de nuestra familia y amigos, de la escuela, y en nuestra vida profesional de los colegas, de los pacientes que atendemos y las comunidades en donde estos viven. Damos por sentado la superioridad de nuestras organizaciones sanitarias y nuestros modelos de atención a la salud, respecto a los que dispone el Sur emergente ${ }^{14,15}$, pero en realidad, no conocemos comparaciones en relación al perfil del MdF, y una forma útil de lograrlo es a través de las narraciones. 
Defensiva. La medicina de familia está preparada para enfrentarse a las amenazas (áreas, actividades o servicios en los que no se considera líder):

- En las actividades docentes e investigadoras

- En el sistema de registro clínico poco seguro
Supervivencia. La medicina de familia se enfrenta a amenazas externas sin las fuerzas internas necesarias para luchar contra ellas. Es aconsejable dejar las cosas tal y como están hasta que se asienten los cambios que se producen:

- Se hace trabajo de grupo pero no trabajo en equipo

- La formación continuada es autoformación

- Difícil conciliación de la vida personal y laboral

- Incomprensión de la medicina de familia

- Presencia de actividades administrativas
Ofensiva. La medicina de familia debe adoptar una estrategia de crecimiento (se reconocen las fortalezas y se resaltan las ventajas propias):

- El amplio rango de actividades del médico de familia latinoamericano

- El trabajo hospitalario del médico de familia

- La asistencia a comunidades donde una mayoría de la población atendida son mujeres

- Las experiencias de asistencia en contextos multiétnicos de clase social baja o con población emigrante o minorías marginadas

- El aprendizaje respecto a las actividades comunitarias y la participación de la familia fuera del centro de salud

- La sensibilidad cultural

- La medicina tradicional

- El aprendizaje en habilidades de comunicación y de la relación médicopaciente con sensibilidad cultural

- $\quad$ El logro de mayor satisfacción laboral

- El aprendizaje de como trabajar con recursos limitados, con flexibilidad y adaptabilidad

- La reflexión sobre una cosmovisión indígena que la armonía entre los individuos y de éstos con la naturaleza

Reorientación. Aparecen oportunidades que se pueden aprovechar, pero la medicina de familia carece de la preparación adecuada. Son las oportunidades de Latinoamérica para aprender de España sobre:

- Estrategias para afrontar con otros ojos las demandas aparentemente insaciables

- Sobre la sensibilidad cultural sobre emigrantes europeos

- A integrar algunas actividades de prevención, promoción, educación para la salud y comunidad en la asistencia en el centro de salud

- A la asistencia de enfermedades crónicas no transmisibles

- A lograr una mejor organización para reducir las dificultades de las largas jornadas de trabajo, especialmente en las zonas rurales más agrestes y duras, y a evitar el pluriempleo.

- A buscar mecanismos para gestionar la difícil accesibilidad en zonas rurales

- La carencia de recursos puede dar lugar a una estrategia de intercambios académicos entre los países. 
Defensiva. La medicina de familia está preparada para enfrentarse a las amenazas (áreas, actividades o servicios en los que no se considera líder):

- En las actividades docentes e investigadoras

- En el sistema de registro clínico doble por carencias del oficial (con un sistema oficial y otro personal) en España

Supervivencia. La medicina de familia se enfrenta a amenazas externas sin las fuerzas internas necesarias para luchar contra ellas. Es aconsejable dejar las cosas tal y como están hasta que se asienten los cambios que se producen:

- Se hace trabajo de grupo pero no trabajo en equipo

- La formación continuada es autoformación

- Difícil conciliación de la vida personal y laboral

- Incomprensión de la medicina de familia

- Presencia de actividades administrativas
Ofensiva. La medicina de familia debe adoptar una estrategia de crecimiento (se reconocen las fortalezas y se resaltan las ventajas propias):

- Afrontar con otros ojos las demandas aparentemente insaciables

- Integrar algunas actividades de prevención, promoción, educación para la salud y comunidad en la asistencia en el centro de salud

Reorientación. Aparecen oportunidades que se pueden aprovechar, pero la medicina de familia carece de la preparación adecuada. Son las oportunidades de España para aprender de Latinoamérica sobre:

- El amplio rango de actividades del médico de familia latinoamericano

- Del trabajo hospitalario del médico de familia

- De la asistencia a comunidades donde una mayoría de la población atendida son mujeres

- De las experiencias de asistencia en contextos multiétnicos de clase social baja o con población emigrante o minorías marginadas

- En el aprendizaje respecto a las actividades comunitarias y la participación de la familia fuera del centro de salud

- Sobre la sensibilidad cultural

- Sobre la medicina tradicional

- En el aprendizaje en habilidades de comunicación y de la relación médicopaciente

- En la asistencia de enfermedades infecciosas

- En el logro de mayor satisfacción laboral

- En el aprendizaje de como trabajar con recursos limitados, con flexibilidad y adaptabilidad 
Las historias narradas en los diarios de un día normal ayudan a explicar por qué las cosas suceden de una determinada forma; dan orden y significado a los eventos -un aspecto crucial del entendimiento de las posibilidades futuras. La narración proporciona significado y perspectiva ${ }^{16}$.

¿Existen, entonces, lecciones que el Norte puede aprender del Sur sobre el perfil profesional del MdF? ¿Y el Sur del Norte? ¿Cuáles podrían ser esas lecciones posibles? El análisis DAFO es un método sencillo y eficaz para decidir sobre el futuro como herramienta estratégica. Nos ayuda a plantear las acciones que deberíamos poner en marcha para aprovechar las oportunidades detectadas y a preparar a nuestra organización ${ }^{11}$, y ha sido ampliamente usado también en el campo sanitario, incluida la planificación de programas formativos ${ }^{12,17,18}$. Nosotros encontramos "fortalezas" del Sur (Paraguay, México y Perú) y del Norte (España) y cómo podían emerger ciertas estrategias: defensiva, de supervivencia, ofensiva (a partir de las fortalezas) y de reorientación (las oportunidades a aprovechar), para Latinoamérica y para España (tablas 3 y 4).

España y Latinoamérica podrían aprender mutuamente en muchas áreas (tabla 5), y nuestros resulta- dos ya han sido esbozados por otros autores ${ }^{19-23}$. Se han descrito las competencias interrelacionadas prioritarias de la educación para la salud global del siglo XX, y para su logro es importante el intercambio formativo entre países ${ }^{24,25}$. La oportunidad de los MdF de un país al trabajar en otro o con MdF de ese otro país, puede lograr aprendizajes para la prestación de la MF a pacientes de otras culturas, incluyendo comunicación y atención culturalmente sensible ${ }^{26}$. En este sentido, se han comunicado la utilidad del intercambio de programas formativos Norte-Sur, pero suelen referirse a aportes del Norte hacia el Sur subdesarrollado que precisa ser formado y ayudado. La integración de la mutualidad o asociación entre países durante los procesos de aprendizaje-enseñanza e investigación tienen un gran beneficio mutuo, pudiéndose beneficiar los profesionales e investigadores de los países con más recursos y menos recursos logrando un fortalecimiento de capacidades en ambos ${ }^{7,27,28}$.

Desde luego, los valores están cambiado y los problemas de salud también. Cada país debe tener su modelo sanitario. Pero, el Sur, debería ser considerado como un factor de aprendizaje para el Norte ${ }^{29}$, porque incluso nos podríamos preguntar si existen

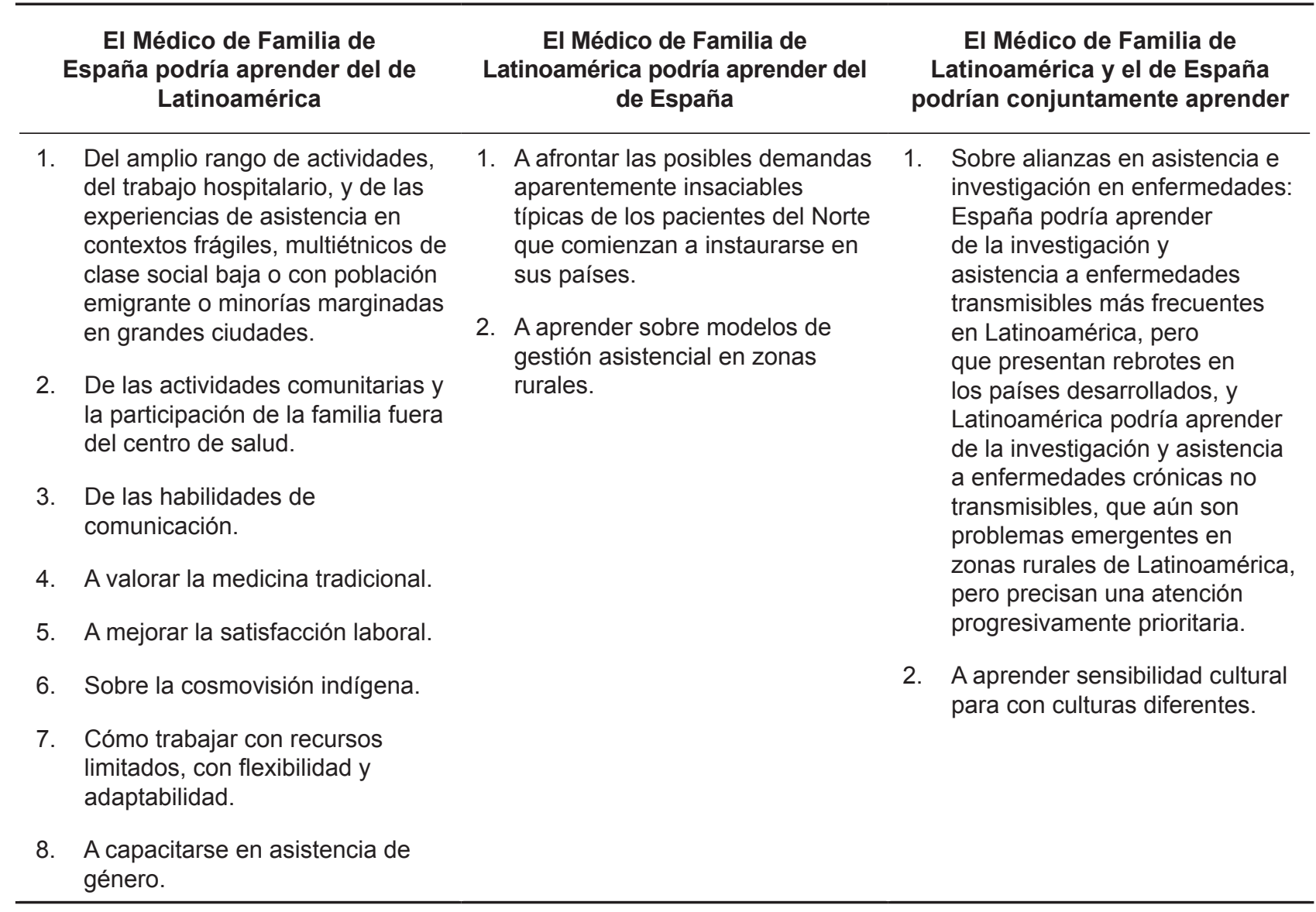

Tabla 5. Áreas donde podría aprender uno del otro, el médico de familia español y el latinoamericano 
los países en desarrollo, o es que están los países desarrollados en camino de ser subdesarrollados.

Como posibles limitaciones de nuestro estudio, además de las ya comunicadas sobre el método de diarios de campo y la técnica de muestreo ${ }^{13}$, hay que señalar que algunos autores han llamado la atención sobre la falta de adecuación del análisis DAFO en el ámbito sanitario europeo, y han presentado otros modelos alternativos ${ }^{18}$. Otra limitación es que no se obtienen los datos en España de las 17 comunidades autónomas existentes, y en cada una de ellas la forma de trabajar del médico de familia puede diferir; y lo mismo ocurre en los distintos países del centro y sur de América, aunque creemos que el estudio aporta un valor explorativo útil, pero no exhaustivo.

En resumen, los países del Norte desarrollado podrían aprender de los países del Sur en desarrollo y viceversa en un amplio rango de actividades del MdF, y sería adecuada la rotación de MdF de países en desarrollo por países desarrollados y viceversa, no para "llevar ayuda", sino para "coger su ayuda".

Como líneas futuras de investigación podemos señalar: el extender y replicar el estudio con diferentes metodologías en más países Latinoamericanos y en desarrollo de otras partes de mundo; el extender el uso de la herramienta cualitativa DAFO en la investigación en MF, y valorar su factibilidad y utilidad para caracterizar fortalezas de los sistemas sanitarios y programas formativos; y desarrollar métodos normalizados para comunicar los beneficios de la colaboración entre países.

\section{AGRADECIMIENTOS}

Los autores de este estudio agradecen el trabajo de los médicos que han participado realizando el diario de un día normal de trabajo.

\section{BIBLIOGRAFÍA}

1. McKee M. Seven goals for public health training in the 21st century. Eur J Public Health. 2013;23:186-187.

2. Haq C, Ventres W, Hunt V, Mull D, Thompson R, Rivo M, et al. Where there is no family doctor: the development of family practice around the world. Acad Med. 1995;70(5):37080.

3. Martineau F, Johnson O, Rowson M, Willott C, Yudkin JS. International health graduates-career path experience. The Lancet. 2012;379(9831):2051-2052.

4. Well C, Rosser WW. Improving health care globally: a critical review of the necessity of family medicine research and recommendations to build research capacity. Ann Fam Med. 2004;26;2(Suppl 2):S5-16.
5. Giordano J. Ethical considerations in the globalization of medicine - an interview with James Giordano. BMC Medicine. 2013,11:69.

6. Waitzkin H, Iriart C, Estrada A, Lamadrid S. Social Medicine Then and Now: Lessons From Latin America. Am J Public Health. 2001;91(10):1592-1601.

7. Redman-MacLaren M, MacLaren DJ, Harrington H, Asugeni R, Timothy-Harrington R, Kekeubata E, Speare R. Mutual research capacity strengthening: a qualitative study of two-way partnerships in public health research. Int J Equity Health.2012,11:79.

8. Adam T, Hsu H, de Savigny D, Lavis JN, Rottingen JA, Bennett $\mathrm{S}$. Evaluating health systems strengthening interventions in low-income and middle-income countries: are we asking the right questions? Health Policy Plan. 2012;27 Suppl 4:iv9-19.

9. Swanson RC, Cattaneo A, Bradley E, Chunharas S, Atun $\mathrm{R}$, Abbas KM, Katsaliaki K, et al. Rethinking health systems strengthening: key systems thinking tools and strategies for transformational change. Health Policy Plan. 2012;27 Suppl 4:iv54-iv61.

10. Martínez Martínez MA. Casos prácticos de Management estratégico. Madrid: Díaz de Santos S.A.; 1996. p. 207-226.

11. McNeilly M. Sun Tzu and the art of business. Six strategic principles for managers. New York: Oxford University Press; 1966. p.144-5.

12. Van Wijngaarden JD, Scholten GR, van Wijk KP. Strategic analysis for health care organizations: the suitability of the SWOT-analysis. Int J Health Plann Manage. 2012; 27(1):3449.

13. Báez-Montiel BB, Gutiérrez-Islas E, Bolaños-Maldonado M, Samarín E, Turabián JL. Las odiseas de los Ulises. Estudio de los relatos de un día normal de trabajo del médico de familia en Paraguay, México, Perú y España. Aten Primaria. 2013. En Prensa. http://dx.doi.org/10.1016/j. aprim.2013.07.003

14. Ventres WB. Cultural encounters and family medicine: six lessons from South America. JABFP. 1997;10:232-236.

15. Elbers W, Schulpen L Corridors of Power: The Institutional Design of North-South NGO Partnerships. Voluntas. International Journal of Voluntary and Nonprofit Organizations 2013; 24 (1):48-67. Disponible en: http://springer.r.delivery.net/r/r?2.1.Ee.2Tp. XARIh.C $4 \% 2$ anA O..T.ZRd \% $5 \mathrm{f} .40 \mathrm{KQ}$. bW89MQ\%5f\%5fCJOAFKY0 [Consultado el 3/02/2014]

16. Greenhalgh T, Hurwitz B (Editors). Narrative based medicine. Dialogue and discourse in clinical practice. London: BMJ Books; 1998.

17. Berlanga Fernández S, Pérez Cañaveras RM, Vizcaya Moreno MF, Torres Rusiñol M, González López NA. Reflexiones sobre la actividad grupal en Atención Primaria. Aten Primaria. 2012;44(11):686-7.

18. Caruana CJ, Wasilewska-Radwanska M, Aurengo A, Dendy PP, Karenauskaite V, Malisan MR, et al. A comprehensive SWOT audit of the role of the biomedical physicist in the education of healthcare professionals in Europe. Phys Med. 2010 26(2):98-110.

19. Ventres W. Answers to US health care issues from other countries. Fam Med. 2011;43(8):592-3.

20. Syed SB, Dadwal V, Rutter P, Storr J, Hightower JD, Gooden $\mathrm{R}$, et al. Developed-developing country partnerships: 
Benefits to developed countries? Global Health. 2012, 8:17.

21. Rosenberg E, Richard C, Lussier MT, Abdool SN. Intercultural communication competence in family medicine: lessons from the field. Patient Educ Couns. 2006;61(2):236-45.

22. Ventres W. The joy of family practice. Ann Fam Med. 2012;10(3):264-8.

23. Yamey G. What are the barriers to scaling up health interventions in low and middle income countries? A qualitative study of academic leaders in implementation science. Global Health. 2012;8:11.

24. Pfeiffer J, Beschta J, Hohl S, Gloyd S, Hagopian A, Wasserheit J. Competency-Based Curricula to Transform Global Health: Redesign With the End in Mind. Acad Med. 2013;88(1):131-136.
25. McKee M. Seven goals for public health training in the 21 st century. Eur J Public Health. 2013;23:186-187.

26. Kraeker C, Chandler C. We Learn From Them, They Learn From Us: Global Health Experiences and Host Perceptions of Visiting Health Care Professionals. Acad Med. 2013;88(4):483-487.

27. Van Dormael M, Dugas S, Diarra S. North-South exchange and professional development: experience from Mali and France. Fam Pract. 2007;24:102-107.

28. Svenson N. Research and development in Central America: panorama and prospects for international cooperation. Higher Education. 2013;65(5):661-676.

29. Editorial. Iberoamérica: de lecciones aprendidas y buenas prácticas. Semergen. 2013;39(3):121-2. 